\title{
Multi-Temperature Zone, Droplet-based Microreactor for Increased Temperature Control in Nanoparticle Synthesis
}

\author{
E. Yegân Erdem, * Jim C. Cheng, Fiona M. Doyle, and Albert P. Pisano
}

Printable electronics and the increased use of nanoparticle products (e.g. quantum dots) has resulted in the rapid development of nanoparticle inks. Microfluidic reactors - or microreactors - show promise to provide advanced control over size, size distribution and shape for commercial-scale synthesis of nanoparticles. Compared to batch-wise synthesis techniques primarily in use today, microfluidic technology can provide better control of the reaction conditions, which is the key to controlling the product characteristics. ${ }^{[1]}$ Handling small volumes of liquid allows better control of mixing, and hence yields more uniform chemical composition. For thermally activated syntheses, reagents can be heated and cooled rapidly and uniformly, avoiding the large thermal gradients typically found throughout the reaction volume in batch techniques. Several high-temperature microreactors for synthesizing nanoparticles have been described in the literature. ${ }^{[2]}$ Almost all microreactors described to date have a single heated zone where nucleation and growth of particles as well as reaction processes which should be controlled at different temperatures for proper completion, are executed

\section{Dr. E. Y. Erdem}

Department of Mechanical Engineering

EA 122, Bilkent University, Ankara, 06800, Turkey

E-mail: yeganerdem@bilkent.edu.tr

Dr. E. Y. Erdem

Department of Mechanical Engineering

Berkeley Sensor and Actuator Center

403 Cory Hall

University of California

Berkeley, 94720, CA, USA

Dr. J.C. Cheng

Department of Electrical Engineering

Berkeley Sensor and Actuator Center

403 Cory Hall

University of California

Berkeley, 94720, CA, USA

Prof. F. M. Doyle

Department of Materials Science \& Engineering

210 Hearst Memorial Mining Building

University of California

Berkeley, 94720, CA, USA

Prof. A. P. Pisano

Department of Mechanical and Aerospace Engineering

7313 Jacobs Hall, University of California

San Diego, CA, 92093

DOI: $10.1002 / \mathrm{smll} .201302379$ at the same temperature. In order to obtain monodisperse or near-monodisperse size distributions, the nucleation and growth stages must be separated. ${ }^{[3]}$ LaMer et al. discussed this concept for the reduction of thiosulphate to sulphur, in terms of the concentration of sulphur monomers. ${ }^{[4]}$ The rate at which thiosulphate is reduced, and hence the sulphur monomer concentration at a given time, is temperature dependent. The principles elucidated by LaMer are broadly applicable to thermally activated syntheses. Of the few reactors with multiple temperature zones, Yang et al. introduced a capillary-based microreactor. ${ }^{[21]}$ However due to the lack of thermal isolation between these two zones, it was not possible to quench nucleation. This paper focuses on the design and fabrication of an ideal, functioning Multi-Temperature zone Microreactor (MTM) on a silicon substrate, built upon theoretical concepts introduced by Winterton et al. ${ }^{[5]}$ As discussed by Winterton et al., the necessary concentration profiles can be controlled by controlling the temperature as a function of time. ${ }^{[5]}$ Ideally, one needs a very short zone of the reactor at a high temperature, to trigger nucleation, followed by a longer duration at a lower temperature where growth occurs to ensure narrow nanoparticle distributions. However Winterton et al. did not fabricate a microreactor to base their ideas. The MTM is the first fabricated and experimentally tested microreactor in literature that has multiple thermally isolated heated and cooled zones designed to separate nucleation and growth as well as to provide a platform for carrying out a systematic study on the effects of temperature and residence time on nanoparticle formation. In this work, $\mathrm{TiO}_{2}$ nanoparticles were selected as a model system to demonstrate the functionality of the microreactor.

The MTM utilizes two-phase flow, requiring a region for passive generation of droplets, in addition to thermallyisolated heated and cooled regions. A two-phase flow profile provides uniform residence times for given fluid elements throughout their passage through the reactor, and prevents deposition of nanoparticles on the channel walls, with attendant disruption of flow. This increases monodispersity of the overall system. An image of the MTM is shown in Figure 1. The microreactor is fabricated in a silicon substrate and sealed by an anodically bonded Pyrex wafer on top. The microchannels are $200 \mu \mathrm{m}$ in width and depth. There are three reaction zones that can be heated independently and the rest of the microreactor is cooled by a cooling channel to quench reactions when solvents exit the reaction zones. These heated and cooled zones are thermally isolated from each 


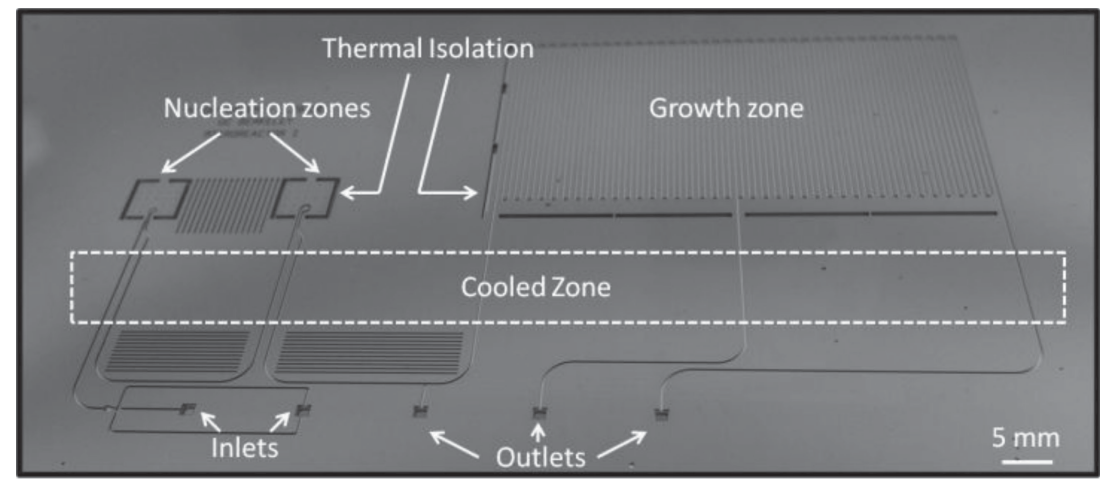

Figure 1. Image of the MTM. There are three thermally isolated zones for heating and one zone for cooling.

other by deep reactive ion etched isolation gaps of $0.5 \mathrm{~mm}$ around the zones and held in place by small tethers through which the channels pass. Figure 2 shows the schematic of a detailed view of these zones. Two of the reaction zones are relatively short $(3 \mathrm{~mm})$. These were designed as the nucleation zones for high temperature reactions. These two zones have different channel widths to offer two different residence time options for a given flow rate. In general, only one nucleation zone would be active at a given time. The third zone is longer $(2000 \mathrm{~mm})$ than the nucleation zones and can be used for slower processes, such as growth of nuclei. This growth zone was designed with two different outlets, to allow discharge of product from either the middle or the end of the zone, thereby offering two different possible growth times. These outlets could also be used to inject additional reagents, such as ligands or stabilizers, or even additional reactants for forming hybrid nanocomposite particles. In this work, however, they were used to discharge the reaction mixture at different stages for characterization. The thermally isolated reaction zones were heated by external, high thermal flux ceramic heaters and a cooling channel was utilized to quench the reactions outside of the reaction zones. Quenching the

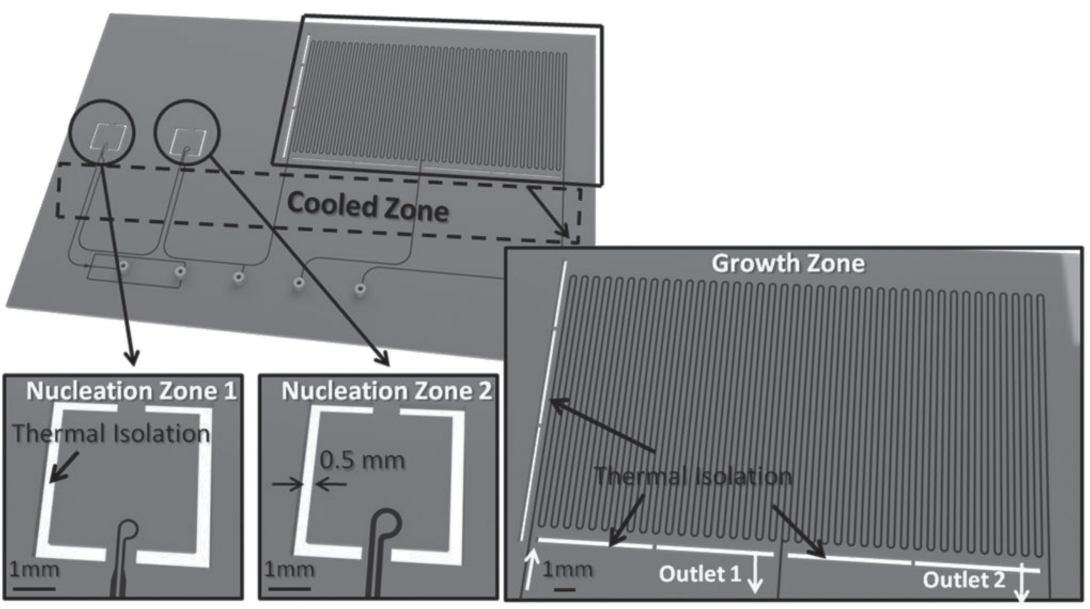

Figure 2. Details of the nucleation and growth zones. The two nucleation zones have the same length of microchannel but different widths, $100 \mu \mathrm{m}$ and $200 \mu \mathrm{m}$ in the first and second nucleation zones, respectively. The growth zone comprised a much longer channel, $200 \mu \mathrm{m}$ wide. reactions as soon as the reagents exit the reaction zones is important to control residence times and temperatures. Temperatures at the reaction zones were monitored by using an infrared (IR) camera as well as thermocouples at the heated zones for comparison. Due to its smaller size, the nucleation zone was able to stably reach its desired temperature within a very short period of 10 seconds whereas the growth zone was able to reach the desired temperature in 15-20 s based on our observation from the IR camera. In a general bulk reactor, modification of the temperatures could take tens of minutes if not longer prior to reaching temperature stability. During the reaction temperatures at the heated zones were constant. An IR camera image demonstrating the temperature of the microreactor is shown in Figure 3. This image shows the sharp temperature difference between the heated and cooled zones and it shows that nucleation and growth zones are isolated. Out gassing or boiling was not observed within the range of temperatures used in the microreactor. Even though the droplet phase was aqueous, there was no gas bubble formation due to the high pressure inside the microchannel. The residence time inside the heated zones were calculated based on the flow rate of the carrier fluid which was determined by a software controlling the syringe pumps delivering the fluids to the reactor. Also the flow speed of droplets was monitored with a high speed camera.

For stable, continuous operation, droplet generation should be stable and the droplet size should be uniform. The carrier fluid and the droplet phase are delivered to the microreactor by syringe pumps. Droplets within an immiscible carrier fluid are generated by using a triangular channel geometry to form uniform and stable droplets following the method in Tan et al. ${ }^{[6]}$ The droplet generation region is shown schematically in Figure 4A. Where the two phases meet, the carrier phase impinges on the continuous stream of dispersed phase, applying sufficient shear stress to pinch the stream into droplets. This geometry ensures that there is only one maximum point of stress within the generation unit where the break-up of droplets can occur. Figure 4B shows the numerical analysis of this phenomenon, it demonstrates that there is a maximum point of velocity (therefore stress) at the narrowest region of the triangular microchannel. Without the tapered design, flow rate after the meeting point of fluids would be uniform and droplet formation would not be guaranteed to occur at the same location at each time. Figure $4 \mathrm{C}$ and Figure 4D show the real time images of the formation of droplets and droplets in growth zone respectively. Typical droplet size was in the range of $10^{7} \mu \mathrm{m}^{3}$ based on our calculations from the high speed 


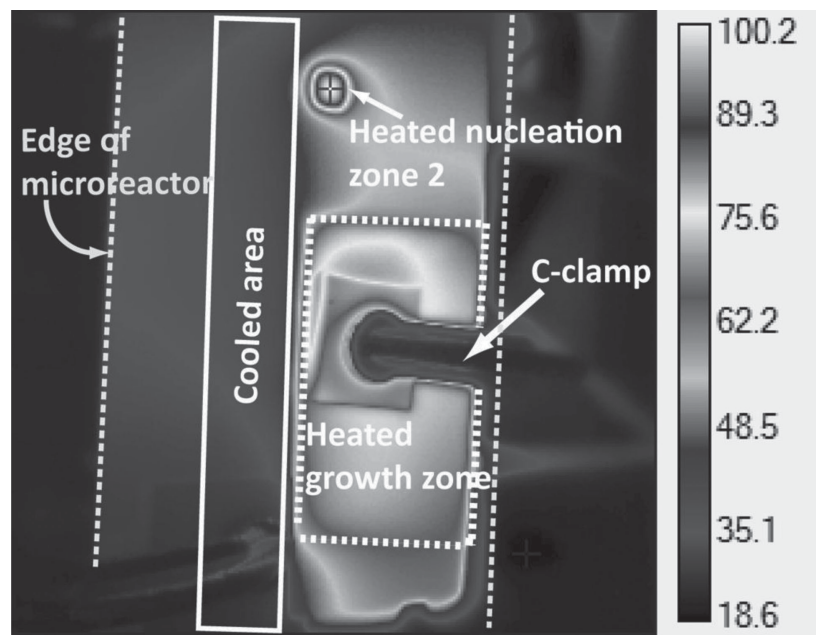

Figure 3. IR camera measurement of the heated and cooled zones. The sharp change of temperature on the chip can be observed. The reagents pass through the cooled area before entering the next heated zone (growth zone) therefore the temperature above $50^{\circ} \mathrm{C}$ between the nucleation and growth is not significant as there is not a channel in that area. (The dark square in the growth zone is the gasket used to allow for distributed mechanical contact from clamp to the reactor.)

camera images. The microchannels were not treated to be made hydrophobic. The topping layer was a Pyrex wafer, which is hydrophilic and the rest was silicon dioxide (due to the oxidation of the silicon wafer), which is hydrophilic. On the other hand, a surfactant, Span 80, was mixed inside the carrier fluid to stabilize the droplet formation as well as to prevent aqueous droplet phase from contacting the channel walls. Therefore stable formation of droplets were observed without surface treatment.

We demonstrated the synthesis of $\mathrm{TiO}_{2}$ nanoparticles inside the microreactor by following the method of Cassaignon et al. ${ }^{[7]}$ The hydrolysis reactions that create $\mathrm{TiO}_{2}$ nanoparticles are very sensitive to temperature, $\mathrm{pH}$ and solution concentration. In this study the solution chemistry was kept constant and the impact of temperature and residence time on the resulting nanoparticles were explored. The hydrolysis reactions responsible for the formation of $\mathrm{TiO}_{2}$ proceed via the formation of hydroxo-bridged oligomers that grow, dehydrate, and gradually increase in crystallinity. Unless there is a significant driving force, such as high $\mathrm{pH}$, hydrolysis reactions are slow at ambient temperature. The MTM affords the opportunity to observe the impact of temperature and time on particle characteristics.

Three different temperature and residence time combinations were probed. In all these experiments the cooled region was kept below $30^{\circ} \mathrm{C}$ to prevent any reaction to occur outside of reaction zones. As a first step the nucleation zone 2 was heated to $100{ }^{\circ} \mathrm{C}$. The flow rate was controlled to provide a $6 \mathrm{~s}$ residence time in the nucleation (short) zone. A TEM image of material extracted immediately after nucleation is shown in Figure 5A. The $\mathrm{TiO}_{2}$ particles appear sheet-like, not yet in a discrete form, and irregularly agglomerated. Next the nucleation zone was again held at $100{ }^{\circ} \mathrm{C}$ but the fluids passed through the first half of the growth zone, held at $80^{\circ} \mathrm{C}$, before discharge. The residence time was $6 \mathrm{~s}$ in the nucleation zone and $120 \mathrm{~s}$ in the growth zone. Figure $5 \mathrm{~B}$ shows the product. It is apparent that holding for $120 \mathrm{~s}$ at $80{ }^{\circ} \mathrm{C}$ did, indeed allow the particles to grow, apparently thicken, and become more uniform. Finally, both the nucleation and growth zones were held at $100{ }^{\circ} \mathrm{C}$, and the solutions passed through them had a total residence time of 240 s. These particles are shown in Figure $5 \mathrm{C}$. These are more uniform than those in Figure 5B, more compact, and more ordered (see inset). The average particle diameter is $26.5 \pm 1.6 \mathrm{~nm}$.

This study exemplifies the utility of the microfluidic device for studying the evolution of nanoparticles. In addition, the device shows significant promise for manufacturing of nanoparticles; the controlled temperature and residence times yielded particles with a standard deviation of size of $10 \%$ better than that achieved in batch-wise methods that used the same chemistry. ${ }^{[7]}$ Through use of parallelism and droplet arraying, future work can explore optimizing the MTM for largescale manufacture of nanoparticles.

In conclusion we have designed and fabricated a microreactor for synthesizing nanoparticles that incorporates multiple thermally-isolated zones. To the best of our knowledge, this is the first microreactor that is capable of separating nucleation and growth by having multiple 


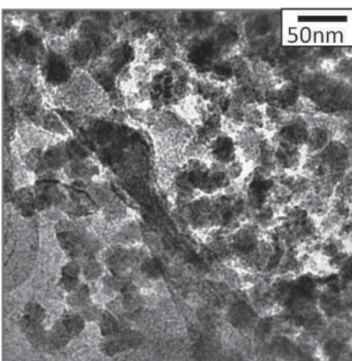

(A)

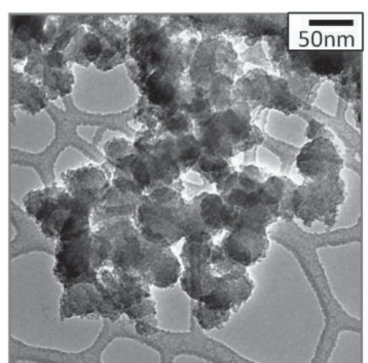

(B)

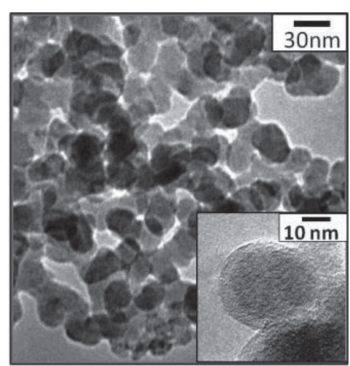

(C)

Figure 5. (A) TEM image of nanoparticles after heating at $100{ }^{\circ} \mathrm{C}$ for $6 \mathrm{~s}$ in the second nucleation zone. (B) TEM image of nanoparticles after heating at $100^{\circ} \mathrm{C}$ for $6 \mathrm{~s}$ in the second nucleation zone and $80^{\circ} \mathrm{C}$ for $120 \mathrm{~s}$ in the first half of the growth zone. (C) TEM image of nanoparticles after heating at $100^{\circ} \mathrm{C}$ for $240 \mathrm{~s}$.

thermally-isolated heated and cooled regions as well as the first platform that gives ability to conduct a detailed study the effects of temperature profile and residence time combinations. Through studies of $\mathrm{TiO}_{2}$ hydrolysis we have demonstrated how this microreactor can be used to make a detailed study of the effects of different temperatures and thermal histories on nanoparticle synthesis. Various other nanoparticle synthesis reactions could also be performed in this reactor.

\section{Experimental Section}

Reagents and Materials: Titanium (III) chloride solution (10 wt.\% in 20-30 wt.\% hydrochloric acid, Sigma-Aldrich) and sodium hydroxide (1N, Fisher Scientific) were used to prepare the aqueous reagent solution (dispersed phase). Octadecane and Span 80 was obtained from Sigma-Aldrich and were used to prepare the carrier phase. All chemicals were used without any further purification and deaeration steps.

Fabrication of the Microreactor: Microreactor was fabricated on a silicon substrate in the Marvell Nanofabrication Facility at UC Berkeley. Two different chrome masks were used in the fabrication. The first mask defines the microchannels; the second mask defines the thermal isolation trenches. In order to ensure thermal isolation, through etching of silicon around the heated zones is required. Thermal isolation and microchannels were formed by deep reactive ion etching of silicon. At the end of the fabrication process, Pyrex wafer is anodically bonded to the silicon substrate to encapsulate microchannels.

In deep reactive ion etching, the etch rate is higher in larger surfaces exposed to the plasma. Since the growth region has a larger surface area than other parts of the microreactor, parallel lines, which can be seen in Figure 1, were added in relatively less dense parts of the design. These lines were disconnected to the microchannel where the reaction occurred. Schematic of the fabrication steps are shown in Figure S1.

Experimental Set-Up: Experimental set-up of the microreactor system consists of the microreactor, ceramic heaters for heating, the cooling channel, water cooling system to deliver cold water to the cooling channel, a high-speed camera for observation and imaging, two syringe pumps to deliver reagents, two power supplies for ceramic heaters, thermocouples for temperature measurements, a thermocouple reader and a frame that holds the microreactor such that it only contacts the heating and cooling units. We have also used thermo paste to prevent any air gap between surfaces and clamped on the substrate. Thermocouples were attached to the heated zones to monitor the temperatures. Microreactor was cooled with an aluminium block with an engraved fluid channel through which cooled water is passed. The Al block makes contact with the silicon surface of the microreactor close to the heated regions, such that it quenched fluid emerging from the heated zones, to suppress nucleation or growth outside of the heated zones, assuring well defined residence times at a given temperature.

The inlet and outlet fluidic interconnects were purchased from LabSmith and bonded onto the microreactor with an epoxy. Silica capillary tubing (also purchased from LabSmith) was used to deliver reagents from glass syringes to the microreactor.

Synthesis of $\mathrm{TiO}_{2}$ Nanoparticles Using the Microreactor: $\mathrm{TiO}_{2}$ nanoparticles were synthesized inside the microreactor via hydrolysis and oxidation of $\mathrm{TiCl}_{3}$ by following the method of Cassaignon et al. ${ }^{[7]}$ The hydrolysis reactions that create $\mathrm{TiO}_{2}$ nanoparticles are very sensitive to temperature, $\mathrm{pH}$ and solution concentration. In this study the solution chemistry was kept constant, and the impact of temperature and residence time on the resulting nanoparticles was studied. The aqueous reagent was prepared by partially neutralizing $0.15 \mathrm{M} \mathrm{TiCl}_{3}$ solution (in 20-30 wt.\% hydrochloric acid) using $0.25 \mathrm{M} \mathrm{NaOH}$ to a $\mathrm{pH}$ of $0.5 .{ }^{[7]} \mathrm{A} 2 \mathrm{wt} \%$ solution of Span 80 in octadecane was used as the carrier phase.

The reaction product was collected at the outlets of the microreactor in a small beaker. After the synthesis, the collected sample was centrifuged with a 2:1 mixture of acetone and ethanol to remove the octadecane with surfactant and to disperse the nanoparticles in acetone. The centrifuge was done by using acetone and ethanol with a mixing ratio of 2:1 multiple times at $4000 \mathrm{rpm}$ for $15 \mathrm{~min}$. Nanoparticles were eventually dispersed in acetone.

Preparation of $\mathrm{TiO}_{2}$ Nanoparticles for Characterization: TEM was used for characterization of nanoparticles. A droplet of nanoparticle solution was placed on the carbon grid and after the acetone which is the solvent they were dispersed in evaporated; the grid was taken into the TEM.

\section{Supporting Information}

Supporting Information is available from the Wiley Online Library or from the author. 


\section{Acknowledgements}

E. Y. Erdem would like to acknowledge Jane Lewis Fellowship (UC Berkeley, Department of Material Science \& Engineering). Authors are grateful to King Abdullah University of Science and Technology (KAUST) for funding this project.

[1] a) A. J. deMello, A. deMello, Lab. Chip. 2004, 4, 11N; b) A. J. deMello, Nature 2006, 442, 394; c) C.-H. Chang, B. K. Paul, V. T. Remcho, S. Atre, J. E. Hutchison, J. Nanopart. Res. 2008, 10, 965; d) A. Abou-Hassan, O. Sandre, V. Cabuil, Angew. Chem. Int. Ed. 2010, 49, 6268; e) R. L. Hartman, K. F. Jensen, Lab. Chip. 2009, 9, 2495; f) S. Marre, K. F. Jensen, Chem. Soc. Rev. 2009, 39, 1183; g) A. M. Nightingale, J. C. deMello, J. Mater. Chem. 2010, 20, 8454; h) A. M. Nightingale, J. C. deMello, Adv. Mat. 2013, 25, 1813; i) Y. Song, J. Hormes, C. S. S. R. Kumar, Small 2008, 4, 698.

[2] a) A. L. Abdelhady, M. Afzaal, M. A. Malik, P. O'Brien, J. Mater. Chem. 2011, 21, 18768; b) E. M. Chan, R. A. Mathies, A. P. Alivisatos, Nano Lett. 2003, 3, 199; c) E. M. Chan, A. P. Alivisatos, R. A. Mathies, J. Am. Chem. Soc. 2005, 127, 13854; d) S. Marre, A. Adamo, S. Basak, C. Aymonier, K. F. Jensen,
Ind. Eng. Chem. Res. 2010, 49, 11310; e) A. M. Nightingale, S. H. Krishnadasan, D. Berhanu, X. Niu,C. Drury, R. McIntyre, Lab. Chip. 2011, 11, 1221; f) B. K. H. Yen, A. Gunther, M. A. Schmidt, K. F. Jensen, M. G. Bawendi, Angew. Chem. 2005, 117, 5583; g) S.-K. Lee, X. Liu, V. S. Cabeza, K. F. Jensen, Lab. Chip. 2012, 12, 4080; h) X. Z. Lin, A. D. Terepka, H. Yang, Nano Lett. 2004, 4, 2227; i) S. Gomez-de Pedro, C. S. Martinez-Cisneros, M. Puyol, J. Alonso-Chamarro, Lab. Chip. 2012, 12, 1979; j) H. Nakamura, Y. Yamaguchi, M. Miyazaki, H. Maeda, M. Uehara, P. Mulvaney, Chem. Commun. 2002, 23, 2844; k) V. S. Cabeza, S. Kuhn, A. A. Kulkarni, K. F. Jensen, Langmuir 2012, 28, 7007; l) H. Yang, W. Luan, S. Tu, Z. M. Wang, Lab. Chip. 2008, 8, 451; m) B. F. Cottam, S. Krishnadasan, A. J. deMello, J. C. deMello, M. S. P. Shaffer, Lab. Chip. 2007, 7, 167.

[3] M. Hosokawa, K. Nogi, M. Naito, T. Yokoyama, Nanoparticle Technology Handbook, Elsevier, Amsterdam, Netherlands 2007.

[4] V. K. LaMer, R. H. Dinegar, J. Am. Chem. Soc. 1950, 72, 4847.

[5] J. D. Winterton, D. R. Myers, J. M. Lippmann, A. P. Pisano, F. M. Doyle, J. Nanopart. Res. 2008, 10, 893.

[6] Y.-C. Tan, V. Cristini, A. P. Lee, Sens. Act. B. 2006, 114, 350.

[7] S. Cassaignon, M. Koelsch, J.-P. Jolivet, J. Phys. Chem. Solids 2007, 68, 695 .

Received: August 4, 2013

Revised: September 27, 2013

Published online: December 12, 2013 